\title{
Electroacupuncture Enhances Antioxidative Signal Pathway and Attenuates Neuropathic Pain Induced by Chemotherapeutic Paclitaxel
}

\author{
Xiaoming ZHAO ${ }^{1^{*}}$, Li LIU ${ }^{1^{*}}$, Yuku WANG ${ }^{2}$, Gang WANG $^{3}$, Yingkai ZHAO ${ }^{4}$, Yao ZHANG \\ * These authors contributed equally to this work. \\ ${ }^{1}$ Tumor Center, The First Hospital of Jilin University, Changchun, Jilin, China, ${ }^{2}$ Jilin Heart Disease \\ Hospital, Changchun, Jilin, China, ${ }^{3}$ Division of Operation, The First Hospital of Jilin University, \\ Changchun, Jilin, China, ${ }^{4}$ Department of Gerontology, The First Hospital of Jilin University, \\ Changchun, Jilin, China, ${ }^{5}$ Department of Thoracic Surgery, The First Hospital of Jilin University, \\ Changchun, Jilin, China
}

Received November 15, 2018

Accepted January 25, 2018

Epub Ahead of Print March 22, 2019

\section{Summary}

One of the significant limiting complications of paclitaxel is painful peripheral neuropathy during its therapy for several types of cancers. Our recent study showed that impairment of Nrf2-antioxidant response element (Nrf2-ARE) and upregulation of oxidative signals in the dorsal root ganglion (DRG) of rats with treatment of paclitaxel result in neuropathic pain. The purpose of this study was to examine the beneficial role played by electroacupuncture (EA) in modifying neuropathic pain evoked by paclitaxel via Nrf2-ARE and oxidative mechanisms. Behavioral test was performed to determine mechanical and thermal sensitivity in rats. Western Blot analysis and ELISA were used to examine expression of Nrf2-ARE and superoxide dismutases (SOD); and the levels of products of oxidative stress in the DRG. Our data showed that paclitaxel increased mechanical and thermal sensitivity and this was accompanied with impaired Nrf2-ARE and SOD in the DRG and amplified products of oxidative stress (i.e. 8-isoprostaglandin F2a and 8-hydroxy-2'deoxyguanosine). EA treatment largely restored the levels of Nrf2-ARE/SOD and inhibited products of oxidative stress and thereby attenuated mechanical and thermal hypersensitivity induced by paclitaxel. In conclusion, we revealed specific signaling pathways leading to paclitaxel-evoked neuropathic pain, including impairment of Nrf2-ARE and heightened oxidative signals. We further provided evidence for the role of EA in alleviating paclitaxel-neuropathic pain via these molecular mediators.

\section{Key words}

Electroacupuncture • Paclitaxel • Neuropathic pain • Nrf2-ARE • $\mathrm{SOD} \bullet$ Oxidative stress

\section{Corresponding authors}

Y. Zhao and Y. Zhang, Department of Gerontology and Department of Thoracic Surgery, The First Hospital of Jilin University, 71 Xinmin Street, Changchun, Jilin 130021, China. E-mail: ykz9798@163.com

\section{Introduction}

Paclitaxel is generally used for the treatment of breast, lung and other cancers, but causes a variety of serious side effects, including peripheral neuropathy, leukopenia, joint or muscle pain, vomiting and alopecia (Ghersi et al. 2015). Chemotherapy-induced peripheral neuropathy causes severe sensory disturbances that range from mild tingling to spontaneous painful burning paresthesia affecting the sensory nerves to the hands and feet (Dougherty et al. 2004) and can persist long after treatment cessation (Tanabe et al. 2013) in cancer patients with chemotherapy (Seretny et al. 2014). As traditional analgesics generally lack efficacy in treating this condition (Kim et al. 2015), it is necessary to have a novel alternative approach for analgesic strategies.

It has been suggested that paclitaxel alters the excitability and survival of neurons via a number of 
mechanisms, which include disruption of axonal transport, impairment of mitochondrial, amplification of ion channel activity as well as neuronal injury and inflammation (Sisignano et al. 2014). In particular, previous studies demonstrated that paclitaxel upregulates multiple transcription factors, enzymes, and cytokines such as interleukin-1 $\beta$ (IL-1 $\beta$ ), interleukin-6 (IL-6) and tumor necrosis factor-alpha (TNF- $\alpha)$, playing an important role in regulating neuropathic and inflammatory pain (Cata et al. 2008). These inflammatory mediators develop neuropathic pain through activation of the Janus-activated kinase-signal transducer and activator of transcription (JAK/STAT) transduction pathway as well as activation of mitogen-activated protein kinase (MAPK) pathway (Miller et al. 2009). In addition, oxidative stress and reduction of nerve growth factor have been involved in paclitaxel-induced neuropathic pain (Griffiths and Flatters 2015).

Nuclear factor (erythroid-derived 2)-like 2 (Nrf2) is a transcription factor and as a basic leucine zipper protein it regulates the expression of antioxidant proteins protecting against oxidative damage triggered by injury and inflammation (Moi et al. 1994). Numerous drugs that stimulate the Nrf2 pathway were used for treatment of diseases caused by oxidative stress (Gold et al. 2012). In a prior study (Miao et al. 2018), we suggested the engagement of antioxidative signal in the dorsal root ganglion (DRG) in paclitaxel-induced neuropathic pain. This prior study specifically showed that paclitaxel impaired Nrf2-antioxidant response element (Nrf2-ARE) and superoxide dismutases (SOD, a class of enzymes to catalyze the dismutation of superoxide) in the DRG and amplified products of oxidative stress. Systemic administration of SOD mimetic and antioxidant vitamin $\mathrm{C}$ or NADPH oxidase (NOX) inhibitor attenuated mechanical and thermal hypersensitivity induced by paclitaxel. This inhibitory effect was accompanied with decreases of proinflammatory cytokines (PICs) in the DRG.

Electroacupuncture (EA) has been used for the clinical treatment of chronic pain caused by various diseases (Kavoussi and Ross 2007, McDonald et al. 2013). It has also been documented for the analgesic effects of EA stimulation in animal with peripheral nerve injuries and inflammatory and neuropathic pain (Kim et al. 2005, Pittler and Ernst 2008, DeLeo and Yezierski 2001). Thus, it is likely that EA is beneficial for alternative treatment strategy of neuropathic pain during paclitaxel chemotherapy and this requires studies of the underlying molecular mechanisms by which EA improves paclitaxel-induced neuropathic pain.

Accordingly, in this report we hypothesized that EA intervention restores impairment of Nrf2-ARE and Nrf2-regulated NADPH quinone oxidoreductase-1 (NQO1) in the DRG of rats with injection of paclitaxel. We also hypothesized that EA intervention restores the decreased levels of SOD and inhibits oxidative NOX, and thereby attenuates amplification of 8-isoprostaglandin F2 $\alpha$ (8-iso PGF2 $\alpha$, a product of oxidative stress); and 8-hydroxy-2'-deoxyguanosine (8-OHdG, a key biomarker of protein oxidation) in the DRG of paclitaxel rats. In addition, we hypothesized that EA decreases the levels of PICs (IL-1 $\beta$, IL-6 and TNF- $\alpha$ ) in the DRG of rats with injection of paclitaxel.

\section{Methods}

Animal

All animal protocols were in accordance with the guidelines of the International Association for the Study of Pain and approved by the Institutional Animal Care and Use Committee of Jilin University. Adult male Sprague-Dawley rats $(200-250 \mathrm{~g})$ were housed in individual cages with free access to food and water and were kept in a temperature-controlled room $\left(25^{\circ} \mathrm{C}\right)$ on a 12/12 h light/dark cycle.

\section{A model of neuropathic pain and EA intervention}

Paclitaxel (Tocris Biosci) was dissolved in a $40 \%$ dimethyl sulfoxide (DMSO) (Smith et al. 2004, Chen et al. 2011). Neuropathy was induced in rats by intraperitoneal (i.p.) injection of paclitaxel $(1 \mathrm{mg} / \mathrm{kg} / \mathrm{one}$ injection in $0.5 \mathrm{ml}$ of volume) every two days and a total of four injections were given (Smith et al. 2004, Chen et al. 2011). Control rats received four injections of $40 \%$ DMSO $(0.5 \mathrm{ml})$ every two days. Mechanical and thermal sensitivity was tested after paclitaxel injection. Mechanical and thermal hypersensitivity were tested daily after paclitaxel. Mechanical and thermal hypersensitivity were fully developed after four injections of paclitaxel and then six hours of behavioral test was performed as shown in Figure 1A. In separate rats, DRG tissues were removed for western blot analysis and ELISA measurement.

Neiguan and Jianshi acupoints [pericardial meridian (P) 5-6, located on the median nerve] were selected for EA because they represent activation of central regions having inhibitory descending pathways in 
involvement of somatic and visceral nociceptive responses (Zhou Yi Syuu et al. 2006, Lujan et al. 2007, Choi et al. 2012). The unrestrained EA method was used to allow rats to move freely in the cage during EA stimulation. The rats were briefly restrained in plastic tubes with the hind limbs extending through two holes. Hook-shaped needles were promptly inserted into the skin and underneath tissues at acupoints. The rats were then released from tubes and allowed to move freely in the cages with the inserted needles. Stainless needles were bilaterally placed at the Neiguan and Jiangshi acupoint 1.5-2.0 and $2.5-3.0 \mathrm{~mm}$ above the wrist (between the ligaments of the flexor carpi radialis and the palmaris longus), respectively. In general, the criterion for accurate needle positioning is relied on the observation of a slight repetitive flexion of the paw during stimulation ( $\mathrm{Li}$ et al. 2006). The stimulation was set at a low current and low frequency $(0.5 \mathrm{~ms}$ pulses, $2 \mathrm{~Hz}$ and $2 \mathrm{~mA}$ ) by an EA stimulator. This was conducted $20 \mathrm{~min} /$ day for five-seven days before each of the experiments. Sham-stimulation was applied to vehicle control rats and paclitaxel rats.

\section{Behavioral test}

To quantify the mechanical sensitivity of the hind paw, rats were placed in individual plastic boxes and allowed to acclimate for $>30 \mathrm{~min}$. Mechanical paw withdrawal threshold (PWT) of rat hind paw in response to the stimulation of von Frey filaments was determined. A series of calibrated von Frey filaments (ranging from 0.5 to $18.0 \mathrm{~g}$ ) were applied perpendicularly to the plantar surface of the hind paw with a sufficient force to bend the filaments or until paw withdrew. In the presence of a response, the filament of next lower force was applied. In the absence of a response, the filament of next greater force was applied. To avoid injury during tests, the cutoff strength of the von Frey filament was $18 \mathrm{~g}$. The tactile stimulus producing a $50 \%$ likelihood of withdrawal was determined using the "up-down" method (Chaplan et al. 1994). Each trial was repeated 2 times at approximately 2 min intervals. The mean value was used as the force produced a withdrawal response.

To determine thermal hyperalgesia, rat paw withdrawal latency (PWL) to a radiant heat was measured. Rats were placed individually in plastic cages on an elevated glass platform and allowed for $30 \mathrm{~min}$ acclimation. Each hind paw received three stimuli with a 10 min interval, and the mean of the three withdrawal latencies was defined as PWL. The heat was maintained at a constant intensity. To prevent tissue damage, the cut-off latency was set at $20 \mathrm{~s}$. To prevent experimental bias, all the behavioral tests were performed in a blind manner. In all the tests, the experimenter had no knowledge (blinded) about the treatments that the rats had received.

\section{ELISA measurements}

Briefly, DRGs (L4-L6) were removed and total protein of DRG tissues was then extracted by homogenizing sample in ice-cold immunoprecipitation assay buffer. The levels of 8 -iso PGF $2 \alpha$ and $8-\mathrm{OHdG}$ in the DRGs (L4-L6) were examined using an ELISA assay kits (obtained from Promega Co. and Abcam Co.) according to the provided description. Briefly, polystyrene 96-well microtiter immunoplates were coated with affinity-purified rabbit primary antibodies. Parallel wells were coated with purified rabbit IgG for evaluation of nonspecific signal. After overnight incubation, plates were washed. Then, the diluted samples and 8 -iso PGF2 $\alpha / 8-O H d G$ standard solutions $(100 \mathrm{pg} / \mathrm{ml}-$ $100 \mathrm{ng} / \mathrm{ml}$ ) were distributed in each plate. The plates were washed and incubated with anti-8-iso PGF2 $\alpha / 8$ OHdG galactosidase. Then, the plates were washed and incubated with substrate solution. After incubation, the optical density was measured using an ELISA reader.

\section{Western blot analysis}

The lysates were centrifuged and the supernatants were collected. After being denatured by heating at $95{ }^{\circ} \mathrm{C}$ in an SDS sample buffer, the supernatant samples were loaded onto Mini-Protean TGX Precast gels and electrically transferred to a polyvinylidene fluoride membrane. The membrane was then incubated overnight with diluted primary antibodies: rabbit anti-Nrf2 (at 1:250), anti-NQO1 (at 1:500), anti-SOD (at 1:500) and anti-NOX4 (at 1:250). After being washed, the membrane was incubated with horseradish peroxidaselinked anti-rabbit secondary antibody (diluted at 1:500) and visualized for immunoreactivity. All antibodies were obtained from Abcam Co., Santa Cruz Biotech and/or or Antibodies online Com. The membrane was stripped and incubated with anti- $\beta$-actin to show equal loading of the protein. The bands recognized by the primary antibody were visualized by exposure of the membrane onto an X-ray film. Then, the film was scanned and the optical densities of primary antibodies and $\beta$-actin bands were determined using the Scion Software. Values for densities of immunoreactive bands/ $\beta$-actin band from the same 
lane were determined. Each of the values was then normalized to a control sample.

\section{Statistical analysis}

All data were analyzed using a two-way analysis of variance for different time courses between two experimental groups. Values were presented as means \pm standard deviation of mean. For all analyses, differences were considered significant at $P<0.05$. All statistical analyses were performed by using SPSS for Windows version 13.0 (SPSS Inc., Chicago, IL).

\section{Results}

Mechanical and thermal sensitivity

In this study, we examined PWT and PWL in rats with injection of DMSO (40\%) and rats with injection of saline. No significant differences in PWT and PWL were observed between groups. i.e. PWT was $13.8 \pm 2.5 \mathrm{~g}$ in saline group $(\mathrm{n}=6)$ vs. $13.2 \pm 1.9 \mathrm{~g}$ in DMSO group $(n=5)(P>0.05$, saline vs. DMSO); and PWL was $12.8 \pm 2.1 \mathrm{~s}$ in saline group $(\mathrm{n}=6)$ vs. $12.1 \pm 1.7 \mathrm{~s}$ in DMSO group $(n=5) \quad(P>0.05$, saline vs. DMSO). This result suggests that DMSO had minimal effects on pain responses evoked by mechanical and thermal stimulation, and a prior report showed that no significant toxic effects were seen by the similar dose of DMSO (Worthley and Schott 1969).

First, we determined time courses for mechanical and thermal sensitivity in control rats and paclitaxel rats by examining PWT and PWL each day after EA treatment. Figure $1 \mathrm{~B}$ shows that PWT and PWL
A
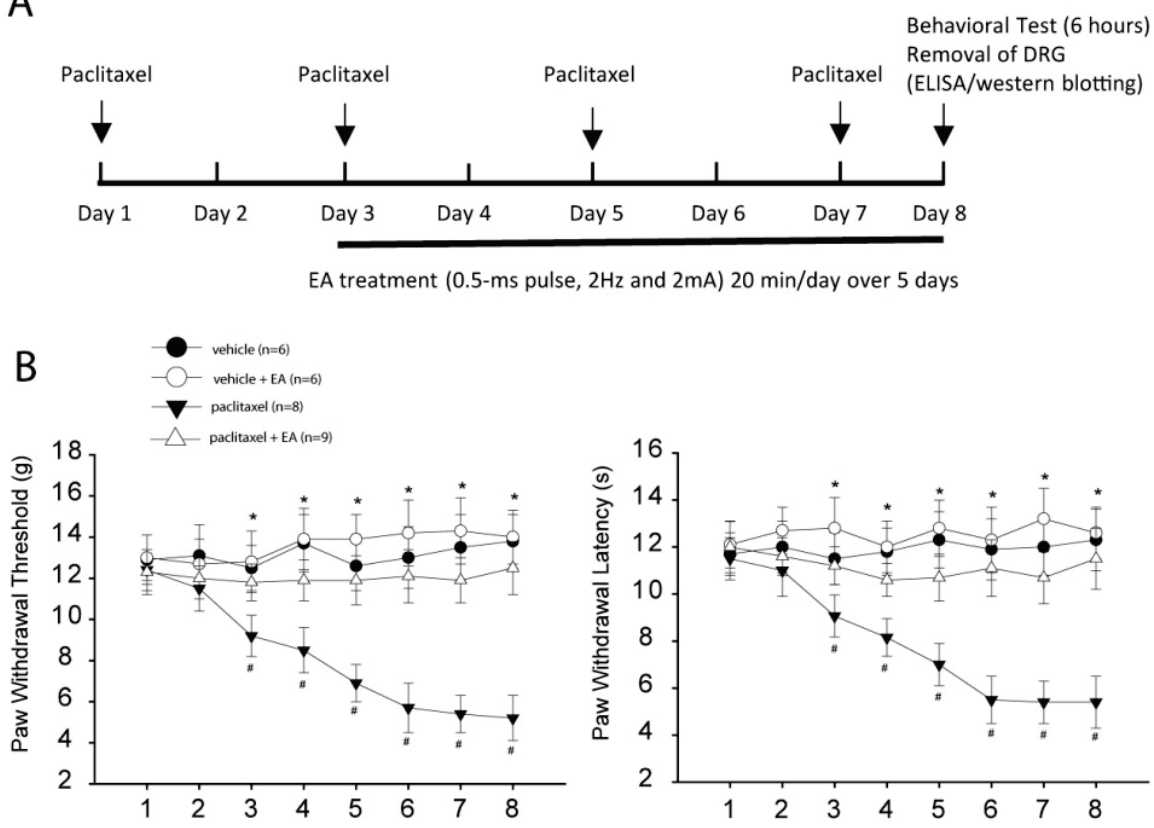

Days after injection of paclitaxel
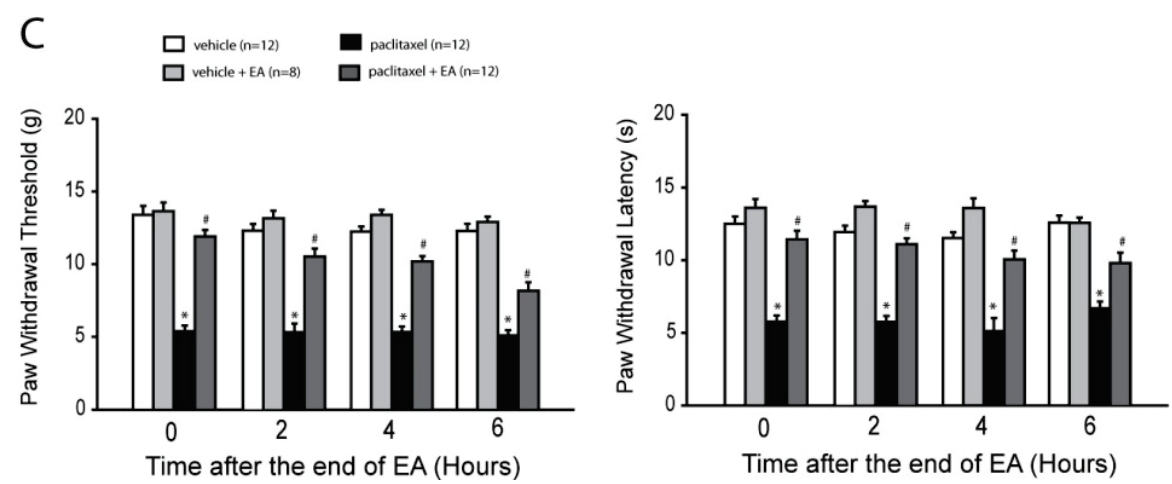

Fig. 1. (A) A schematic diagram showing the schedule for injection of paclitaxel and EA treatment. Paclitaxel was injected [intraperitoneally (i.p.), $1 \mathrm{mg} / \mathrm{kg}$ body weight every other day] (indicated as arrows). An equivalent volume of vehicle was given in control animals. EA was given daily for 5 days. On the $8^{\text {th }}$ day, behavioral tests were performed and in the separate rats, the DRGs were removed for ELISA measurements and western blot analysis. In time course experiment, EA was given daily beginning from day 1. (B) Time courses for mechanical and thermal sensitivity in control rats and paclitaxel rats after EA treatment. Left and right panels indicate the effects of EA on mechanical and thermal sensitivity, respectively. EA increased PWT and PWL during development of mechanical and thermal pain after injection of paclitaxel. $* P<0.05$, paclitaxel rats vs. control rats. ${ }^{\#} P<0.05$, paclitaxel rats vs. paclitaxel rats with EA treatment. The number of animals is indicated on the figure. (C) Mechanical and thermal sensitivity during six hours of test in control rats and paclitaxel rats after EA treatment. Left panel: Effects of EA on mechanical sensitivity in vehicle control rats and paclitaxel rats. As compared with no EA treatment, EA increased PWT in paclitaxel rats. Right panel: Effects of EA on thermal sensitivity in control rats and paclitaxel rats. EA increased PWL in paclitaxel rats. Note that the increases of PWT and PWL evoked by EA treatment were insignificant in control rats. $* P<0.05$ vs. vehicle. 
began to reduce two days after first injection of paclitaxel and they became less with additional paclitaxel injections; EA increased PWT and PWL during development of mechanical and thermal pain after paclitaxel. After four injections of paclitaxel, PWT decreased to a greater degree as compared with vehicle injection $(P<0.05$, paclitaxel rats vs. vehicle control rats; $\mathrm{n}=12$ in each group). A six-hour of behavioral test was performed. Figure 1C (left panel) demonstrates that PWT was increased after EA treatment in paclitaxel rats $(P<0.05$ vs. paclitaxel rats with no EA $n=12$ in each group). In paclitaxel rats, the inhibitory effects of EA on mechanical hypersensitivity lasted for several hours after the end of its treatment. In addition, the increase of PWT evoked by EA was insignificant in vehicle control rats $(P>0.05$ vs. vehicle control rats with no $\mathrm{EA} ; \mathrm{n}=8)$. Likewise, paclitaxel injection significantly diminished PWL as compared with vehicle injection. Figure $1 \mathrm{C}$ (right panel) further shows that EA significantly attenuated thermal hypersensitivity during six hours of test in paclitaxel rats $(P<0.05$ vs. paclitaxel rats with no $\mathrm{EA} ; \mathrm{n}=12$ in each group). The effects of EA intervention on PWL were insignificant in control rats $(P>0.05$ vs. vehicle control rats with no treatment; $n=8$ ).

\section{Expression of Nrf2-ARE and oxidative stress signal pathways}

We first examine the protein levels of $\mathrm{Nrf} 2$ and Nrf2-regulated NQO1 in the DRG. Figure 2A shows that the protein expression levels of Nrf2 and Nrf2-regulated NQO1 were decreased in paclitaxel rats $(P<0.05$, paclitaxel rats vs. control rats; $n=6-10)$. In the similar way, Figure $2 \mathrm{~B}$ further shows that paclitaxel led to downregulation of SOD expression $(P<0.05$, paclitaxel rats vs. controls rats; $n=6-8)$. It is noted that downregulation of Nrf2, NQO1 and SOD in paclitaxel rats were considerably restored after EA treatment as shown in Figure 1A-B $(P<0.05$, paclitaxel rats with EA vs. paclitaxel rats with no $\mathrm{EA} ; \mathrm{n}=6-10)$. In contrast, NOX4 was amplified by paclitaxel and EA significantly attenuated increases of NOX4 as shown in Figure $2 \mathrm{C}$ $(P<0.05$, paclitaxel rats with EA vs. paclitaxel rats with no $\mathrm{EA} ; \mathrm{n}=6-10$ in each group).
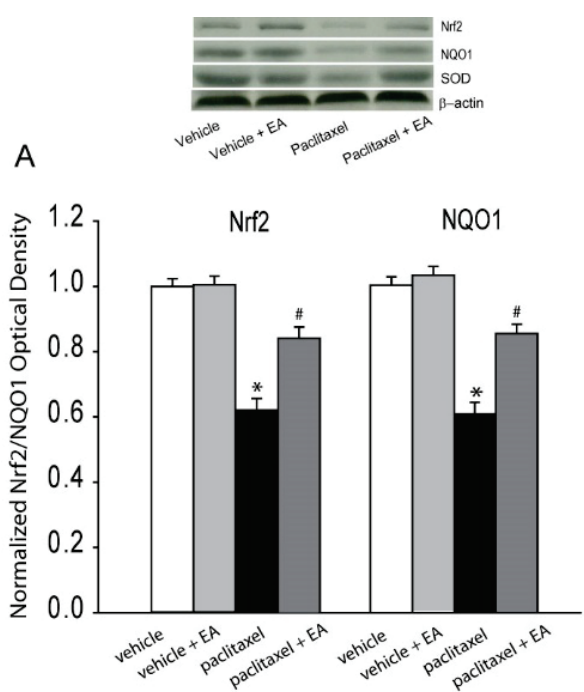

B
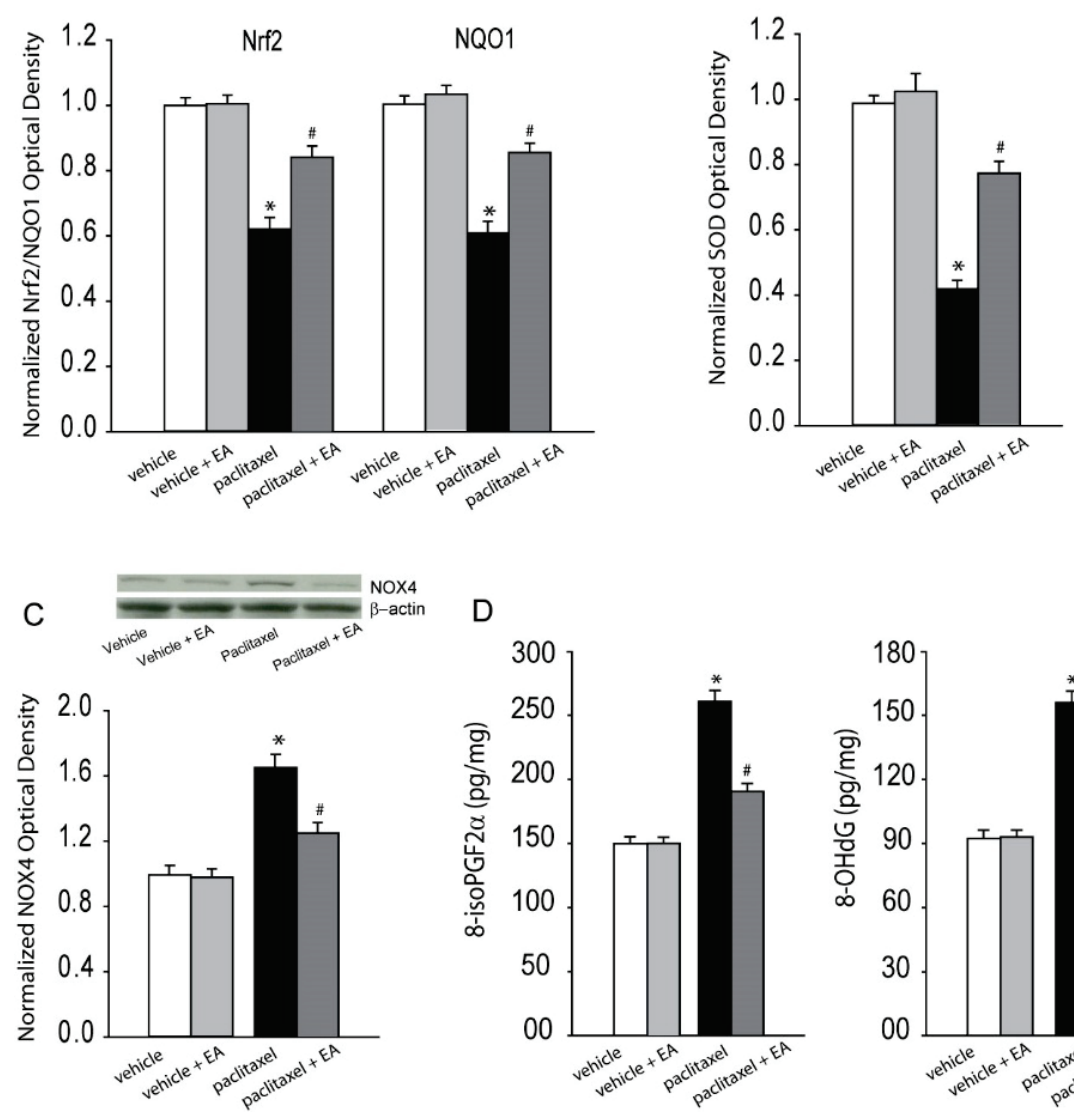
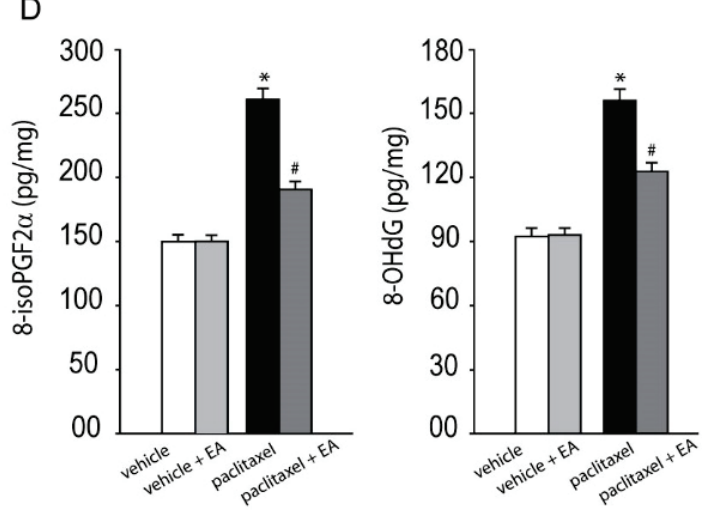

Fig. 2. Effects of paclitaxel on signal pathways of oxidative stress. (A-B) Protein expression levels of Nrf2-ARE and SOD. Top panel and bottom panel are representative bands and averaged data: Nrf2/NQO1 and SOD were decreased in the DRG of paclitaxel rats. $* P<0.05$, paclitaxel rats vs. vehicle control rats. ${ }^{\#} P<0.05$ vs. paclitaxel rats with no $E A . n=6-10$ in each group. (C). Representative bands and averaged data, showing that protein expression levels of NOX4 were amplified by paclitaxel. $* P<0.05$, paclitaxel rats vs. vehicle controls. ${ }^{\#} P<0.05$ vs. paclitaxel rats with no EA. $n=6-10$ in each group. (D) Systemic administration of paclitaxel amplified the levels of oxidative products 8-iso PGF2a and 8 -OHdG in the DRG. $* P<0.05$ vs. vehicle control rats. $\# P<0.05$ vs. paclitaxel rats with no EA. $n=12$ in each group of control rats and paclitaxel rats; $\mathrm{n}=15$. 
In addition, we examined products of oxidative stress in the DRG of control rats and paclitaxel rats as shown in Figure 2D. 8-iso PGF2 $\alpha$ and $8-\mathrm{OHdG}$ were increased in the DRG of paclitaxel rats $(P<0.05$ vs. control rats, $\mathrm{n}=12$ in each group). Note that EA intervention significantly attenuated amplification of 8 -iso PGF2 $\alpha$ and 8 -OHdG induced by paclitaxel $(P<0.05$, paclitaxel rats with $\mathrm{EA} / \mathrm{n}=15$ vs. paclitaxel rats with no $\mathrm{EA} / \mathrm{n}=12$ ).

\section{Levels of PICs}

In additional experiments, we examined the effects of EA on upregulated PICs induced by paclitaxel in the DRG. Figure 3 shows that IL-1 $\beta$, IL- 6 and TNF- $\alpha$ were significantly increased in paclitaxel rats $(n=12$; $P<0.05$ vs. vehicle control rats $/ \mathrm{n}=12$ ) as compared with vehicle control rats. Furthermore, EA intervention $(n=15)$ significantly attenuated amplifications of PICs evoked by paclitaxel. Insignificant difference in the levels of PICs in the DRG was observed between vehicle control rats with EA and vehicle control rats with no treatment $(P>0.05$, EA treatment $/ \mathrm{n}=12$ vs. no EA treatment $/ \mathrm{n}=10)$.

$$
\begin{aligned}
& \square \text { vehicle }(n=12) \\
& \square \text { vehicle + EA (n=10) } \square \text { paclitaxel }(n=12) \\
& \text { paclitaxel + EA ( } n=15)
\end{aligned}
$$
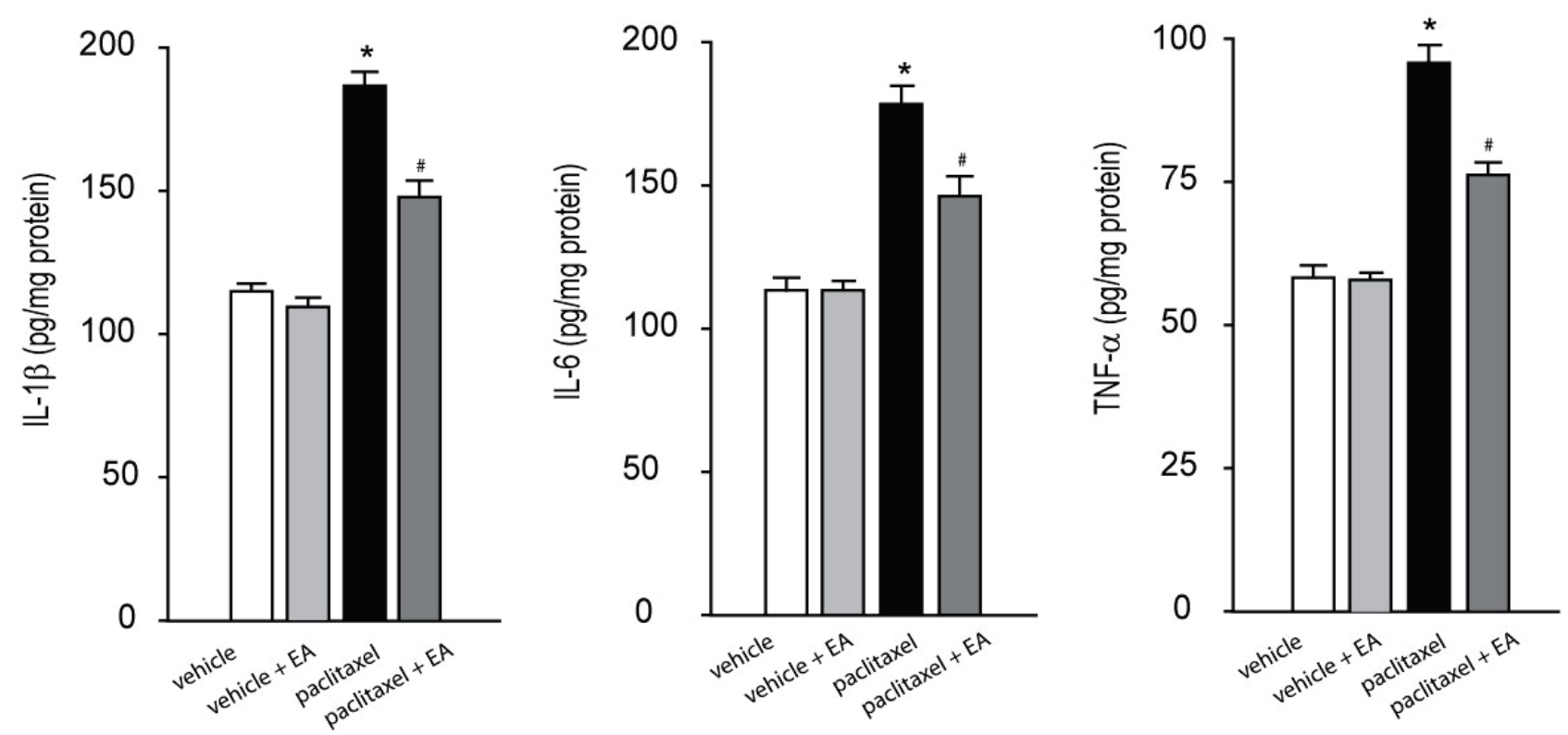

Fig. 3. Levels of PICs in control rat and paclitaxel rats. Paclitaxel increased IL-1 $\beta$, IL-6 and TNF- $a$ as compared with vehicle control rats. EA treatment significantly attenuated amplifications of PICs evoked by paclitaxel. $* P<0.05$ vs. control rats and paclitaxel rats with EA treatment. ${ }^{*} P<0.05$ vs. paclitaxel rats with no EA. $n=12$ in vehicle control; $n=10$ in vehicle control $+E A ; n=12$ in paclitaxel; $n=15$ in paclitaxel + EA.

\section{Discussion}

One of the most common and distressing symptoms suffered by patients with progression of cancer is pain (Hanna et al. 2013). Cancer pain mainly arises from a tumor compressing or infiltrating tissue; from nerve and other changes caused by a hormone imbalance or immune response; and/or from treatments and diagnostic procedures (Pasetto et al. 2006, Hanna et al. 2013). It should be noted that chemotherapy and radiotherapy produce painful conditions persisting long after treatment has ended (Hoskin 2008, Portenoy 2011,
Hanna et al. 2013). As a result, how to effectively manage cancer pain related to these therapies becomes am important issue for treatment and management of cancer patients in clinics. Nonetheless, treatment options for these abnormal sensations have been restricted, partly due to a poor understanding of the underlying mechanisms responsible for neuropathic pain induced by chemotherapy. Also, there are lacking of effective drugs applied to patients; and costs and side effects of drugs affect a treatment strategy of neuropathic pain in patients with chemotherapy. Alternative treatment strategy of neuropathic pain is noteworthy during chemotherapy. 
Thus, in this report we examined the beneficial role play by EA intervention in modifying neuropathic pain induced by chemotherapeutic paclitaxel.

Prior studies have shown that injection of paclitaxel produces neuropathic pain in rats including mechanical hyperalgesia and thermal hypersensitivity several days after initiation of the chemotherapy regimen in rats (Chen et al. 2011). The signs of mechanical hyperalgesia and thermal hypersensitivity lasted for several weeks after administration of paclitaxel (Chen et al. 2011). Thus, in this study we employed this well-established rat model to examine the mechanisms leading to neuropathic pain induced by paclitaxel. Using the same intervention, in our current study we observed that PWT declined to evoke mechanical withdrawal and PWL decreased to induce thermal withdrawal after injection of paclitaxel.

In addition, results of our recent study demonstrated that the expression of Nrf2-ARE signal and SOD expression were downregulated in the DRG of paclitaxel rats as compared with control rats (Miao et al. 2018). The expression of NOX4, the levels of oxidative products 8 -iso PGF2 $\alpha / 8$-OHdG and PICs such as IL- $1 \beta$, IL- 6 and TNF- $\alpha$ were amplified in the DRG of paclitaxel rats. In this prior study (Miao et al. 2018), we further demonstrated that PICs were decreased and mechanical and thermal hypersensitivity were attenuated in paclitaxel rats after inhibition of NOX4 and systemic supplying of SOD and antioxidant vitamin C. Notably, results of current study showed that EA intervention 1) restored impairment of Nrf2-ARE and SOD in the DRG of paclitaxel rats; 2) inhibited amplification of NOX4 and 8-iso PGF2 $\alpha / 8$-OHdG and PICs, thereby alleviating neuropathic pain induced by paclitaxel.

The role of oxidative stress in chemotherapyinduced peripheral neuropathy has been studied. As a non-specific reactive oxygen species (ROS) scavenger, N-tert-butyl- $\alpha$-phenylnitrone (PBN) was reported to inhibit the development of paclitaxel-induced mechanical hypersensitivity (Fidanboylu et al. 2011). Also, tempol inhibited the development and maintenance of paclitaxelinduced mechanical hypersensitivity, but was ineffective on cold allodynia (Fidanboylu et al. 2011, Kim et al. 2017). In addition, the activity of different antioxidant enzymes in the DRG and peripheral sensory nerves were examined during the time course of paclitaxel-induced painful neuropathy. Enhanced activity of mitochondrial and cellular endogenous antioxidant enzymes in the DRG and peripheral nerves was observed, however this was inadequate and delayed in its onset leading to excessive ROS in peripheral sensory axons (Duggett et al. 2016). Others have demonstrated an impaired mitochondrial antioxidant response after paclitaxel (Janes et al. 2013). In our current study, we demonstrated that expression of SOD was decreased in the DRG of paclitaxel rats and EA largely restored impaired SOD and attenuated paclitaxelinduced mechanical and thermal hypersensitivity. These data provided evidence for the role of EA in modifying mitochondrial ROS in the development of paclitaxelinduced peripheral neuropathy.

In addition to antioxidants and/or free radical scavengers, it is also interesting to determine the effects of decreasing ROS generation on paclitaxel-induced mechanical and thermal hypersensitivity. One source of ROS production is the enzyme family of NOX. The rodent genome encodes four genes that contain the catalytic NOX subunit, namely NOX1, NOX2, NOX3, and NOX4 (Altenhofer et al. 2012). This electrontransferring subunit is constitutively inactive in resting cells and generates ROS only upon activation, e.g. after noxious stimuli (Salvemini et al. 2011). While NOX2 activation is predominantly associated with innate immunity mediated host defense and NOX1 with blood pressure control and related vascular mechanisms (Gavazzi et al. 2006, Lam et al. 2010), NOX4 was shown highly expressed under ischemic conditions in the central nervous system, e.g. ischemic stroke (Suzuki et al. 2012). Evidence has also identified activation of NOX4 as a causative factor that contributes to inflammatory or neuropathic pain role in the peripheral nervous system (Kallenborn-Gerhardt et al. 2012). Thus, in the current study we further determined the effects of EA intervention on expression levels of NOX4 in the DRG of paclitaxel rats. We observed that NOX4 was upregulated after administration of paclitaxel and EA attenuated upregulation of NOX4 as well as increases of oxidative 8 -iso PGF2 $\alpha / 8$-OHdG.

Furthermore, paclitaxel was reported to increase the level of IL- $1 \beta$, IL- 6 and TNF- $\alpha$ in the DRG of rats thereby inducing pain behaviors (Kim et al. 2016, Kim et al. 2017). Consistent with these findings, in the current study we observed that paclitaxel amplified IL-1 $\beta$, IL-6 and TNF- $\alpha$ in the DRG. Interestingly, EA attenuated increases in IL-1 $\beta$, IL- 6 and TNF- $\alpha$ after paclitaxel and attenuated mechanical and thermal hypersensitivity. Results of the present report suggest that downregulation of IL- $1 \beta$, IL- 6 and TNF- $\alpha$ in the DRG is involved in the inhibitory effects of EA on mechanical pain and thermal 
hypersensitivity in paclitaxel-induced neuropathy.

It should be noted that Cochrane reviews of acupuncture for a wide range of pain conditions have been published (Lee and Ernst 2011), suggesting that acupuncture is effective for some but not all types of pain. There have also been many clinical trials in humans testing the effectiveness of EA for relieving neuropathic pain. A recent Cochrane review has indicated that there is insufficient evidence to support or refute the use of acupuncture for neuropathic pain whereas more studies are still ongoing (Ju et al. 2017).

Study limitations: First, one concern is that we used the separated group of rats to perform western blot/ELISA experiments and behavioral experiments and the status of those animals might be different with respect to the development of neuropathic pain. It should be noted that those rats received the same treatment of paclitaxel as well as EA which was likely to cause minimal differences in pain response in both groups. Second, in the current study neuropathic pain began two days after the first injection of paclitaxel and with treatment of EA PWT and PWL were increased, suggesting that EA had inhibitory effects on mechanical and thermal pain during development of neuropathy. Our results also showed that neuropathic pain was fully developed in paclitaxel rats on the $8^{\text {th }}$ day and PWT and PWL remained at higher levels in paclitaxel rats with EA while a six-hour of test was performed. However, a study limitation is that later time points showing the duration of the EA effects were not included.

\section{Conclusions}

In the DRG of paclitaxel rats, Nrf2-ARE signal and SOD expression are downregulated and oxidative products and PICs are amplified, leading to mechanical pain and thermal hypersensitivity. EA intervention has a significant inhibitory effect on neuropathic pain induced by paclitaxel, accompanied with restoring the levels of Nrf2-ARE and SOD as well as inhibiting oxidative products and PICs. Nonetheless, our data demonstrated the beneficial role of EA in modifying neuropathic pain induced by paclitaxel. Clarification of the underlying molecular mechanisms for analgesia of EA would also open an opportunity to combine EA with medications to manage and control pain induced by chemotherapy as a potential clinical implication.

\section{Conflict of Interest}

There is no conflict of interest.

\section{Acknowledgements}

We thank all the people who helped during performing all the experiments.

\section{References}

ALTENHOFER S, KLEIKERS PW, RADERMACHER KA, SCHEURER P, ROB HERMANS JJ, SCHIFFERS P, HO H, WINGLER K, SCHMIDT HH: The NOX toolbox: validating the role of NADPH oxidases in physiology and disease. Cell Mol Life Sci 69: 2327-2343, 2012.

CATA JP, WENG HR, DOUGHERTY PM: The effects of thalidomide and minocycline on taxol-induced hyperalgesia in rats. Brain Res 1229: 100-110, 2008.

CHAPLAN SR, BACH FW, POGREL JW, CHUNG JM, YAKSH TL: Quantitative assessment of tactile allodynia in the rat paw. J Neurosci Methods 53: 55-63, 1994.

CHEN Y, YANG C, WANG ZJ: Proteinase-activated receptor 2 sensitizes transient receptor potential vanilloid 1, transient receptor potential vanilloid 4 , and transient receptor potential ankyrin 1 in paclitaxel-induced neuropathic pain. Neurosci 193: 440-451, 2011.

CHOI EM, JIANG F, LONGHURST JC: Point specificity in acupuncture. Chin Med 7: 4, 2012.

DELEO JA, YEZIERSKI RP: The role of neuroinflammation and neuroimmune activation in persistent pain. Pain 90: $1-6,2001$.

DOUGHERTY PM, CATA JP, CORDELLA JV, BURTON A, WENG HR: Taxol-induced sensory disturbance is characterized by preferential impairment of myelinated fiber function in cancer patients. Pain 109: 132-142, 2004.

DUGGETT NA, GRIFFITHS LA, MCKENNA OE, DE SANTIS V, YONGSANGUANCHAI N, MOKORI EB, FLATTERS SJ: Oxidative stress in the development, maintenance and resolution of paclitaxel-induced painful neuropathy. Neuroscience 333: 13-26, 2016. 
FIDANBOYLU M, GRIFFITHS LA, FLATTERS SJ: Global inhibition of reactive oxygen species (ROS) inhibits paclitaxel-induced painful peripheral neuropathy. PLoS One 6: e25212, 2011.

GAVAZZI G, BANFI B, DEFFERT C, FIETTE L, SCHAPPI M, HERRMANN F, KRAUSE KH: Decreased blood pressure in NOX1-deficient mice. FEBS Lett 580: 497-504, 2006.

GHERSI D, WILLSON ML, CHAN MM, SIMES J, DONOGHUE E, WILCKEN N: Taxane containing regimens for metastatic breast cancer. Cochrane Database Syst Rev 6: CD003366, 2015.

GOLD R, KAPPOS L, ARNOLD DL, BAR-OR A, GIOVANNONI G, SELMAJ K, TORNATORE C, SWEETSER MT, YANG M, SHEIKH SI, DAWSON KT: Placebo-controlled phase 3 study of oral BG-12 for relapsing multiple sclerosis. New Eng J Med 367: 1098-1107, 2012.

GRIFFITHS LA, FLATTERS SJ: Pharmacological modulation of the mitochondrial electron transport chain in paclitaxel-induced painful peripheral neuropathy. J Pain 16: 981-994, 2015.

HANNA M, ZYLICZ Z: Cancer Pain. Springer-Verlag, London, 2013, 286 p.

HOSKIN PJ: Radiotherapy. In: Clinical Pain Management: Cancer Pain. SYKES N, BENNETT MI, YUAN CS (eds), London: Hodder Arnold, 2008, pp 251-255.

JANES K, DOYLE T, BRYANT L, ESPOSITO E, CUZZOCREA S, RYERSE J, BENNETT GJ, SALVEMINI D: Bioenergetic deficits in peripheral nerve sensory axons during chemotherapy-induced neuropathic pain resulting from peroxynitrite-mediated post-translational nitration of mitochondrial superoxide dismutase. Pain 154: 2432-2440, 2013.

JU ZY, WANG K, CUI HS, YAO Y, LIU SM, ZHOU J, CHEN TY, XIA J: Acupuncture for neuropathic pain in adults. Cochrane Database Syst Rev 12: CD012057, 2017.

KALLENBORN-GERHARDT $\mathrm{W}$, SCHRODER K, DEL TURCO D, LU R, KYNAST K, KOSOWSKI J, NIEDERBERGER E, SHAH AM, BRANDES RP, GEISSLINGER G, SCHMIDTKO A: NADPH oxidase-4 maintains neuropathic pain after peripheral nerve injury. J Neurosci 32: 10136-10145, 2012.

KAVOUSSI B, ROSS BE: The neuroimmune basis of anti-inflammatory acupuncture. Integr Cancer Ther 6: 251-257, 2007.

KIM HK, HWANG SH, ABDI S: Tempol ameliorates and prevents mechanical hyperalgesia in a rat model of chemotherapy-induced neuropathic pain. Front Pharmacol 7: 532, 2017.

KIM HK, HWANG SH, LEE SO, KIM SH, ABDI S: Pentoxifylline ameliorates mechanical hyperalgesia in a rat model of chemotherapy-induced neuropathic pain. Pain Physician 19: E589-E600, 2016.

KIM JH, DOUGHERTY PM, ABDI S: Basic science and clinical management of painful and non-painful chemotherapy-related neuropathy. Gynecol Oncol 136: 453-459, 2015.

KIM SK, PARK JH, BAE SJ, KIM JH, HWANG BG, MIN BI, PARK DS, NA HS: Effects of electroacupuncture on cold allodynia in a rat model of neuropathic pain: mediation by spinal adrenergic and serotonergic receptors. Exp Neurol 195: 430-436, 2005.

LAM GY, HUANG J, BRUMELL JH: The many roles of NOX2 NADPH oxidase-derived ROS in immunity. Semin Immunopathol 32: 415-430, 2010.

LEE MS, ERNST E: Acupuncture for pain: an overview of Cochrane reviews. Chin J Integr Med 17: 187-189, 2011.

LI P, TJEN-A-LOOI SC, LONGHURST JC: Excitatory projections from arcuate nucleus to ventrolateral periaqueductal gray in electroacupuncture inhibition of cardiovascular reflexes. Am J Physiol Heart Cir Physiol 290: H2535-H2542, 2006.

LUJAN HL, KRAMER VJ, DICARLO SE: Electroacupuncture decreases the susceptibility to ventricular tachycardia in conscious rats by reducing cardiac metabolic demand. Am J Physiol Heart Cir Physiol 292: H2550-H2555, 2007.

MCDONALD JL, CRIPPS AW, SMITH PK, SMITH CA, XUE CC, GOLIANU B: The anti-inflammatory effects of acupuncture and their relevance to allergic rhinitis: a narrative review and proposed model. Evid Based Complement Alternat Med 2013: 591796, 2013.

MIAO H, XU J, XU D, MA X, ZHAO X, LIU L: Nociceptive behavior induced by chemotherapeutic paclitaxel and beneficial role of antioxidative pathways. Physiol Res 68: 491-500, 2019. 
MILLER RJ, JUNG H, BHANGOO S, WHITE FA: Cytokine and chemokine regulation of sensory neuron function. In: Handbook of Experimental Pharmacology. CANNING BJ, SPINA DV (eds), Springer, Berlin Heidelberg, 2009, pp 417-449.

MOI P, CHAN K, ASUNIS I, CAO A, KAN YW: Isolation of NF-E2-related factor 2 (Nrf2), a NF-E2-like basic leucine zipper transcriptional activator that binds to the tandem NF-E2/AP1 repeat of the beta-globin locus control region. Proc Natl Acad Sci U S A 91: 9926-9930, 1994.

PASETTO LM, D’ANDREA MR, ROSSI E, MONFARDINI S: Oxaliplatin-related neurotoxicity: How and why? Crit Rev Oncol Hematol 59: 159-168, 2006.

PITTLER MH, ERNST E: Complementary therapies for neuropathic and neuralgic pain: systematic review. Clin J Pain 24: 731-733, 2008.

PORTENOY RK: Treatment of cancer pain. Lancet 377: 2236-2247, 2011.

SALVEMINI D, LITTLE JW, DOYLE T, NEUMANN WL: Roles of reactive oxygen and nitrogen species in pain. Free Radic Biol Med 51: 951-966, 2011.

SERETNY M, CURRIE GL, SENA ES, RAMNARINE S, GRANT R, MACLEOD MR, COLVIN LA, FALLON M: Incidence, prevalence, and predictors of chemotherapy-induced peripheral neuropathy: A systematic review and meta-analysis. Pain 155: 2461-2470, 2014.

SISIGNANO M, BARON R, SCHOLICH K, GEISSLINGER G: Mechanism-based treatment for chemotherapyinduced peripheral neuropathic pain. Nat Rev Neurol 10: 694-707, 2014.

SMITH SB, CRAGER SE, MOGIL JS: Paclitaxel-induced neuropathic hypersensitivity in mice: responses in 10 inbred mouse strains. Life Sci 74: 2593-2604, 2004.

SUZUKI Y, HATTORI K, HAMANAKA J, MURASE T, EGASHIRA Y, MISHIRO K, ISHIGURO M, TSURUMA K, HIROSE Y, TANAKA H, YOSHIMURA S, SHIMAZAWA M, INAGAKI N, NAGASAWA H, IWAMA T, HARA H: Pharmacological inhibition of TLR4-NOX4 signal protects against neuronal death in transient focal ischemia. Sci Rep 2: 896, 2012.

TANABE Y, HASHIMOTO K, SHIMIZU C, HIRAKAWA A, HARANO K, YUNOKAWA M, YONEMORI K, KATSUMATA N, TAMURA K, ANDO M, KINOSHITA T, FUJIWARA Y: Paclitaxel-induced peripheral neuropathy in patients receiving adjuvant chemotherapy for breast cancer. Int J Clin Oncology 18: 132-138, 2013.

WORTHLEY EG, SCHOTT CD: The toxicity of four concentrations of DMSO. Toxicol Appl Pharmacol 15: 275-281, 1969.

ZHOU YI SYUU W, HSIAO I, LIN VW, LONGHURST JC: Modulation of cardiovascular excitatory responses in rats by transcutaneous magnetic stimulation: role of the spinal cord. J Appl Physiol (1985) 100: 926-932, 2006. 